\title{
An articulated pes from a small parvicursorine alvarezsauroid dinosaur from Inner Mongolia, China
}

David W.E. Hone, Jonah N. Choiniere. Qingwei Tan, and Xing Xu

Acta Palaeontologica Polonica 58 (3), 2012: 453-458 doi: http://dx.doi.org/10.4202/app.2011.0127

A near complete and articulated parvicursorine pes from the Campanian Wulansuhai

Formation is described. This pes is referred to the genus Linhenykus and is one of the first foot skeletons to be described for a derived alvarezsaur, providing new information on the first digit of the pes. The evolution of a laterally directed flange of the anterior face of the distal third metatarsal in arctometatarsalian taxa is described and discussed. This flange may have increased stability of the foot during cursorial locomotion and may also provide useful taxonomic and systematic data.

Key words: Dinosauria, Theropoda, maniraptoran, arctometatarsal, Cretaceous, Inner Mongolia.

David W.E. Hone [dwe hone@yahoo.com] School of Biology \& Environmental Sciences, University College Dublin, Dublin 4, Ireland and Institute of Vertebrate Palaeontology and Palaeoanthropology, 10044 Beijing, China; Jonah N. Choiniere [jchoiniere@amnh.org] American Museum of Natural History, Central Park West at 79th Street, New York, New York 10024, USA; Qingwei Tan [firsttan@sina.com] Long Hao Institute of Geology and Paleontology, Hohhot, Inner Mongolia, 010010,China; Xu Xing [xu.xing@ivpp.ac.cn] Key Laboratory of Evolutionary Systematics of Vertebrates, Institute of Vertebrate Paleontology \& Paleoanthropology, 10044 Beijing, China.

This is an open-access article distributed under the terms of the Creative Commons Attribution License (for details please see creativecommons.org), which permits unrestricted use, distribution, and reproduction in any medium, provided the original author and source are credited. 
